\title{
Fast Software Implementation of Binary Elliptic Curve Cryptography
}

\author{
Manuel Bluhm ${ }^{1}$ and Shay Gueron ${ }^{2,3}$ \\ 1 Ruhr University Bochum, Germany \\ 2 University of Haifa, Israel \\ 3 Intel Corporation, Israel Development Center, Israel
}

November 10, 2013

\begin{abstract}
This paper presents an efficient and side channel protected software implementation of point multiplication for the standard NIST and SECG binary elliptic curves. The enhanced performance is achieved by improving the Lòpez-Dahab/Montgomery method at the algorithmic level, and by leveraging Intel's AVX architecture and the pclmulqdq processor instruction at the coding level. The fast carry-less multiplication is further used to speed up the reduction on the newest Haswell platforms. For the five NIST curves over $G F\left(2^{m}\right)$ with $m \in\{163,233,283,409,571\}$, the resulting point multiplication implementation is about 6 to 12 times faster than that of OpenSSL-1.0.1e, enhancing the ECDHE and ECDSA algorithms significantly.
\end{abstract}

Keywords: ECC, binary elliptic curves, point multiplication, pclmulqdq, AVX, ECDH, ECDSA, side channel protection, NIST, Lòpez-Dahab.

\section{Introduction}

All SSL/TLS communications start with a client-server key establishment, based on a public key cryptosystem. The classical protocol uses the RSA algorithm for encryption/decryption of a session key, but it suffers from the following drawback: the confidentiality of the sessions depends directly on the security of the server's private RSA key. Especially motivated by recent disclosures, the current trends are to move to protocols providing (perfect) forward secrecy, such as the authenticated Diffie-Hellman Ephemeral (DHE) key exchange. One approach is to use Elliptic Curve Cryptography (ECC) to efficiently perform this kind of key exchange (ECDHE). Similarly, ECC based signature schemes (ECDSA) become attractive because of their relatively short keys. Thus, software implementations of ECC for the high end server platforms become a target for optimization.

Protocols such as TLS 1.x support elliptic curves over both prime and binary fields, and the important cryptographic libraries include implementations for both types of ECC. For instance, the OpenSSL library that underlies the mod_ssl module of Apache, implements the ECDHE-ECDSA, ECDHE-RSA and 
other key agreement protocols over binary and prime fields. It is interesting to note that from the hardware implementation cost, protocols using binary curves are cheaper to implement than prime field curves, which can be significant for low-end client devices. However, on the software implementation side, binary curves have been less efficient than their prime curves counterparts on high end processors. This was mainly caused by the fast integer multiplication units compared to slow carry-less multiplication. In OpenSSL-1.0.1e for example, the prime curve secp256k1 outperformes it's binary counterpart sect283k1 by several magnitudes.

This paper focuses on software optimization of point multiplication over binary elliptic curves, leveraging the AVX instruction set and the newly optimized carry-less multipliers on high end x86-64 platforms. Targeting performance and security by fast, side channel protected code, we built a library for the underlying binary field arithmetic for the common fields specified by NIST and SECG [17, covering security levels from 80 bits up to 256 bits. Table 1 shows the binary elliptic curves over $G F\left(2^{m}\right)$ (both random and Koblitz curves) that are optimized in this paper. It further displays their field representation and the equivalent bit level security, and (in parentheses) the corresponding RSA key length. On the left column, "1" indicates that the respective curve is standardized by NIST (the National Institute of Standards and Technology); “2” indicates a curve standardized by SECG (Standards for Efficient Cryptography Group); "3" indicates that the curve is part of the Wireless Transport Layer Security (WTLS).

\begin{tabular}{|c|c|c|c|c|}
\hline $\begin{array}{l}\text { Curve } \\
\text { identifier }\end{array}$ & Type & $\mathbf{m}$ & $\begin{array}{c}\text { Bit level security } \\
\text { (Equiv. RSA key length) }\end{array}$ & $\begin{array}{l}\text { Field polynomial } \\
\text { for reduction in } G F\left(2^{m}\right)\end{array}$ \\
\hline $\begin{array}{l}\operatorname{sect} 163 \mathrm{k} 1^{1,2,3} \\
\operatorname{sect} 163 \mathrm{r} 1^{2} \\
\text { sect } 163 \mathrm{r} 2^{1,2}\end{array}$ & $\begin{array}{l}\text { Koblitz } \\
\text { Random } \\
\text { Random }\end{array}$ & 163 & $80(1024)$ & $x^{163}+x^{7}+x^{6}+x^{3}+1$ \\
\hline 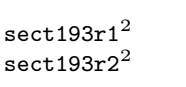 & $\begin{array}{l}\text { Random } \\
\text { Random }\end{array}$ & 193 & $96(1536)$ & $x^{193}+x^{15}+1$ \\
\hline $\begin{array}{l}\operatorname{sect233\mathrm {k}} 1^{1,2,3} \\
\operatorname{sect233r} 1^{1,2,3}\end{array}$ & $\begin{array}{l}\text { Koblitz } \\
\text { Random }\end{array}$ & 233 & $112(2240)$ & $x^{233}+x^{74}+1$ \\
\hline $\operatorname{sect} 239 \mathrm{k} 1^{2}$ & Koblitz & 239 & $115(2304)$ & $x^{239}+x^{158}+1$ \\
\hline $\begin{array}{l}\operatorname{sect} 283 \mathrm{k} 1^{1,2} \\
\operatorname{sect} 283 \mathrm{k} 1^{1,2}\end{array}$ & $\begin{array}{l}\text { Koblitz } \\
\text { Random }\end{array}$ & 283 & $128(3456)$ & $x^{283}+x^{12}+x^{7}+x^{5}+1$ \\
\hline $\begin{array}{l}\operatorname{sect} 409 \mathrm{k} 1^{1,2} \\
\operatorname{sect} 409 \mathrm{r} 1^{1,2}\end{array}$ & $\begin{array}{l}\text { Koblitz } \\
\text { Random }\end{array}$ & 409 & $192(7680)$ & $x^{409}+x^{87}+1$ \\
\hline $\begin{array}{l}\operatorname{sect5} 71 \mathrm{k} 1^{1,2} \\
\operatorname{sect5} 71 \mathrm{r} 1^{1,2}\end{array}$ & $\begin{array}{l}\text { Koblitz } \\
\text { Random }\end{array}$ & 571 & $256(15360)$ & $x^{571}+x^{10}+x^{5}+x^{2}+1$ \\
\hline
\end{tabular}

Table 1: Targeted binary elliptic curves and their security level 


\section{The improved $2 \mathrm{P}$ algorithm}

Given an elliptic curve $\mathcal{E}$ with $|\mathcal{E}|=\left|G_{\mathcal{E}}\right| \cdot\left|H_{\mathcal{E}}\right|=n \cdot h$ (here: $h \in\{2,4\}$ ), the essential operation of elliptic curve cryptosystems is multiplying a point $P \in G_{\mathcal{E}}$ on the curve, by a positive integer $k \in[1, n]$, to obtain a new point

$$
Q=k \cdot P=\underbrace{P+P+\ldots+P}_{\text {k-times }}=\sum_{i=0}^{k-1} P .
$$

Montgomery [15] introduced a fast approach for this point multiplication. It uses the fact that the sum of two points whose difference is known, can be evaluated with a procedure that involves only the $x$-coordinate of these points when performing one point doubling, and one point addition per iteration. López and Dahab (LD hereafter) improved this algorithm for binary curves over $G F\left(2^{m}\right)$, by eliminating costly inversion operations in the field (when given in polynomial representation). In their so-called $\mathbf{2} \mathbf{P}$-Algorithm [14, the point $P=(x, y)$ is represented in the LD-projective form $P=\left(X / Z, Y / Z^{2}\right)$. The $x$-coordinate of the sum $P_{0}+P_{1}$ and of $2 P_{i}$ is the fraction $X^{\prime} / Z^{\prime}$, where

$$
\begin{array}{ll}
\underline{2 P_{i}:} & \underline{P_{0}+P_{1}:} \\
X^{\prime}=X_{i}^{4}+b \cdot Z_{i}^{4} & X^{\prime}=x \cdot Z^{\prime}+\left(Z_{0} \cdot X_{1}\right) \cdot\left(Z_{1} \cdot X_{0}\right) \\
Z^{\prime}=X_{i}^{2} \cdot Z_{i}^{2} & Z^{\prime}=\left(\left(Z_{0} \cdot X_{1}\right)+\left(Z_{1} \cdot X_{0}\right)\right)
\end{array}
$$

The computational cost of the resulting algorithm is dominated by the performance of the point addition(Madd) and point doubling(Mdouble), which are executed $\left\lfloor\log _{2}(k)\right\rfloor$ times in a point multiplication $k P$. Here, we speed up the point multiplication by reducing the amount of computations in Madd and Mdouble.

\subsection{Improved Mdouble}

By re-ordering the flow of the Mdouble, we first multiply $Z^{2}$ with the precomputed value $c=\sqrt{b}$, then add the result to $X^{2}$, square this again and obtain the result $X \leftarrow\left(X^{2}+c \cdot Z^{2}\right)^{2}=X^{4}+b \cdot Z^{4}$. This saves one squaring and one reduction operation per run. Algorithm 2 shows the improved Mdouble flow.

For Koblitz curves, we have $b=1$ and therefore $c=b^{2^{m-1}}=1$, so another multiplication and reduction can be saved, as shown in Algorithm 3 .

\subsection{Improved Madd}

The Madd function can be improved by deferring reduction operations (we call it lazy reduction, after [21]). Algorithm 4 shows the improved Madd flow, where we defer the reduction after Steps 4 and 8, and reduce the result only in Step 9. The rationale is that double sized addition is cheaper than a reduction (the actual gain depends on the specific field). 


\subsection{Estimating the impact of the improved Mdouble and Madd}

Table 2 compares the number of the field operations in Algorithms 2, 3 , and 4 , compared to their original form. For simplicity, we count the reduction as a field operation here.

\begin{tabular}{|c|c|c|c|c|c|}
\hline $\begin{array}{l}\text { Field } \\
\text { Operations }\end{array}$ & $\begin{array}{l}\text { Mdouble } \\
\text { Koblitz }\end{array}$ & $\begin{array}{l}\text { Mdouble } \\
\text { Random }\end{array}$ & $\begin{array}{l}\text { Mdouble } \\
\text { Original }\end{array}$ & $\begin{array}{l}\text { Madd } \\
\text { Improved }\end{array}$ & $\begin{array}{l}\text { Madd } \\
\text { Original }\end{array}$ \\
\hline \#MUL & 1 & $2\left\lfloor\log _{2}(k)\right\rfloor$ & )」 & c)」 & $(k)\rfloor$ \\
\hline & $4\left\lfloor\log _{2}(k)\right\rfloor$ & $\mathbf{5}\left\lfloor\log _{2}(k)\right\rfloor$ & $6\left\lfloor\log _{2}(k)\right\rfloor$ & $4\left\lfloor\log _{2}(k)\right\rfloor$ & $5\left\lfloor\log _{2}(k)\right\rfloor$ \\
\hline & $\mathbf{3}\left\lfloor\log _{2}(k)\right\rfloor$ & $\mathbf{3}\left\lfloor\log _{2}(k)\right\rfloor$ & $4\left\lfloor\log _{2}(k)\right\rfloor$ & $\left\lfloor\log _{2}(k)\right\rfloor$ & $\left\lfloor\log _{2}(k)\right\rfloor$ \\
\hline \#ADD & $\left\lfloor\log _{2}(k)\right\rfloor$ & $\left\lfloor\log _{2}(k)\right\rfloor$ & $\left\lfloor\log _{2}(k)\right\rfloor$ & $\mathbf{3}\left\lfloor\log _{2}(k)\right\rfloor$ & $2\left\lfloor\log _{2}(k)\right\rfloor$ \\
\hline
\end{tabular}

Table 2: The number of field operations for point multiplication, with the original and the improved Mdouble and Madd algorithms.

\section{Side channel protected point multiplication}

This section discusses the potential threat and countermeasures of software side channel attacks, where an adversary attempts to collect information about the secret keys that are being used. Today, it is known that software implementations of cryptographic primitives need to take such side channel attacks into account, and add appropriate countermeasures.

\subsection{Protection requirements}

As with other cryptographic schemes, implementations of ECC have been shown to be susceptible to software side channel attacks. Even the applicability of remote timing attacks on a ECC implementation has been demonstrated [7]. We concentrate here on protecting the software implementation of the LD/Montgomery point multiplication, without covering the higher implementation parts of the various protocols like ECDHE or ECDSA.

Timing attacks exploit the occurrence of variant execution times for different inputs. This kind of attack can be mounted whenever a correlation between the key and the execution time is found. Further, we take cache based timing attacks into account, where the adversary is assumed to be able to run an unprivileged process to measure instruction and data cache latencies. Thus, key dependent branches and table accesses should be avoided or masked.

To avoid timing attacks in the LD/Montgomery point multiplication one must ensure that

1. all inputs $(k, P)$ to the point multiplication function are valid, meaning

- Integer $k$ is in the interval $[1, n]$ with $n=|\langle P\rangle|$

$\circ k$ has constant bit length (for the same group)

$\circ \mathrm{P}$ is a valid point on the curve.

2. the point multiplication and its sub-functions are executed in constant time,

3. no key dependent code branches or data accesses exist. 


\section{$3.2 \quad$ Protection strategy}

The large variety of attacks makes it very difficult to protect an implementation against all possible side channel threats. In the following, we will show the mechanisms to prevent the previously mentioned threats for server implementations.

Constant key length is a first step to assert a constant running time of the point multiplication $k P$. Although, the input validation of the scalar $k$ and the point $P$ should be performed prior to the point multiplication, we provide a bit length fix for all valid $k \in[1, n]$ with $n$ order of the large subgroup $G_{\mathcal{E}}$ of $\mathcal{E}$. At first, we always add $n$ to $k$, so that $k^{\prime}=k+n$ and $k P=k^{\prime} P$. The bit length of $k^{\prime}$ is then $\left\lfloor\log _{2}(n)\right\rfloor \leq \log _{2}\left(k^{\prime}\right) \leq\left\lfloor\log _{2}(n)\right\rfloor+1$, since the result of an addition of two integers $n$ and $k \leq n$ is not longer than $\left\lfloor\log _{2}(n)\right\rfloor+1$ bits.

To make the bit length fixed for all cases of $k$, one can repeat the addition of $n$, if and only if the bit at position $\left\lfloor\log _{2}(n)\right\rfloor+1$ is NOT set. To ensure that the implementation does not include any branches, one should create a mask in a similar way as suggested in Algorithm 1 and then add the masked value, which is either the order $n$ or an equivalent sized number with zero value. Thereafter, the bit length of $k^{\prime}$ is fixed to $\left\lceil\log _{2}(n)\right\rceil$. One has to take into account that $k$ needs to be a valid input with $k \in[1, n]$ in order to assert the fixed bit length, which is expected to be evaluated outside the point multiplication. Additionally, this countermeasure eventually adds vulnerability to Carry-based attacks [8], attacking not the point multiplication but this particular countermeasure itself. However, considering our attacker model this is no reasonable threat.

Constant memory access pattern and the elimination of key dependent branches are the main countermeasure against cache based side channel attacks in our implementation. Since cache attacks are most likely with our attacker model, we want to assure not only a fixed memory access pattern but also avoid any key dependent branch. Therefore, we suggest algorithm 1 to veil the data access.

Let $t x_{1}, t x_{2}, t z_{1}$ and $t z_{2}$ be fixed size temporary variables for a $G F\left(2^{m}\right)$ and $k_{i}$ the current bit at position $i$. Table 3 shows the transition of the coordinates involved in the double/add process inside the LD/Montgomery point multiplication's key evaluation loop.

For the proposed data veiling method, a masking word $t_{0}$ is created with either all bits set to zero, or all bits set to one, depending on the value of $k_{i}$. This word is then used to create the mask for field elements in step 2, which in turn is successively applied with AND and NAND operations to the two possible values, satisfying the rules in Table 3. This transition is self-inverse and needs to be re-applied after the execution of Madd and Mdouble.

\begin{tabular}{c|c|c|} 
& $k_{i}=0$ & $k_{i}=1$ \\
\hline$t x_{1}$ & $x_{2}$ & $x_{1}$ \\
$t x_{2}$ & $x_{1}$ & $x_{2}$ \\
\hline$t z_{1}$ & $z_{2}$ & $z_{1}$ \\
$t z_{2}$ & $z_{1}$ & $z_{2}$ \\
\hline
\end{tabular}

Table 3: X/Z Transformation 


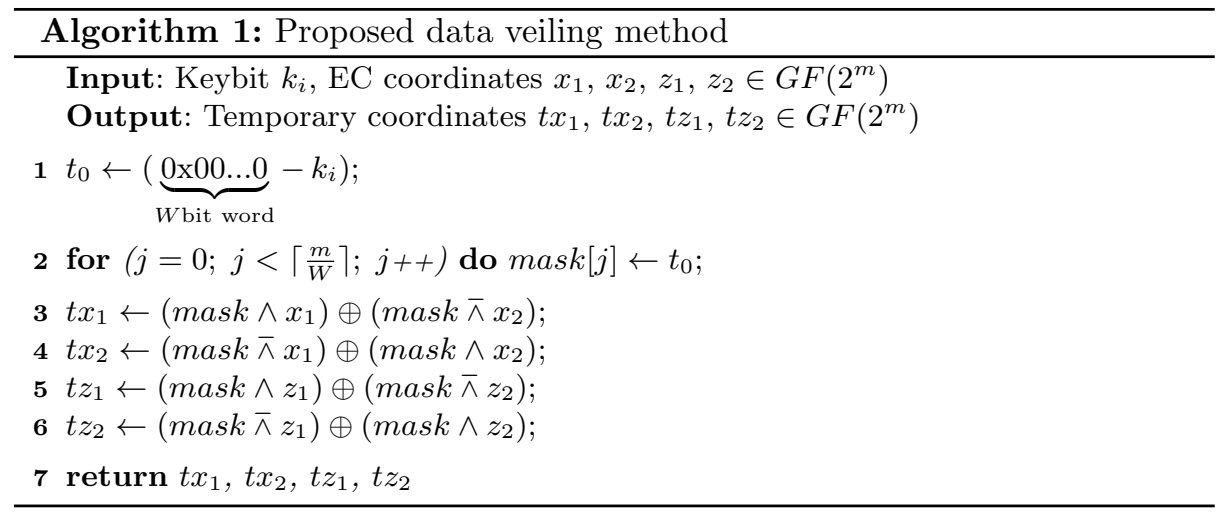

In summary, the proposed technique requires 4 field XORs, NANDs and ANDs, and, neglectable in proportion, one 64 bit subtraction and one constant load each.

Constant function calls inside the key evaluation loop are naturally provided by the LD/Montgomery point multiplication. In addition, we must ensure that all called subfunctions are executed in constant time and thus not include data depending branches. However, due to the branches inside Mxy, which is the final transformation to extract the $y$-coordinate, the running time for the point multiplication is not constant if the result is invalid, the inverse $-P$ of a point $P$ or the point at infinity (and thus the scalar $k$ a multiple of $n$ ).

Constant time implementation for binary field arithmetic is the necessary consequence from the previous point. If we want to ensure that the running time is constant, the binary field arithmetic must be totally data independent. This does not only mean that we cannot include any shortcuts for special values and data dependent branches at all, but it also biases the choice of algorithm, e.g., the inversion method.

\section{Efficient implementation of binary field arithmetics}

In this section we explain the techniques for the implementation of the binary field arithmetic. With special focus on fast reduction, we introduce a reduction scheme using the pclmulqdq instruction to speed up the reduction on the new Haswell architecture. 


\subsection{Binary field arithmetic}

The square of a field element $a(x) \in G F\left(2^{m}\right)$, rather than just multiplying a field element with itself, can be computed as follows:

$$
a^{2}(x)=\left(\sum_{i=0}^{m-1} a_{i} x^{i}\right)^{2}=\sum_{i=0}^{m-1}\left(a_{i} x^{i}\right)^{2}=\sum_{i=0}^{m-1} a_{i} x^{2 i} \bmod f(x)
$$

This property makes squaring in $G F\left(2^{m}\right)$ a linear operation and therefore much faster than the conventional multiplication, and can be performed with small table lookups. This table can be stored in only one vector and is therefore naturally accessed in a fixed pattern. Expanding the element by successively inserting zeros, it can even be computed in parallel, using the pshufb instruction. The use of pshfub to implement lookup tables was originally proposed by Gueron and Kounavis [9] and first used by Aranha et al. in [4] for squaring in $G F\left(2^{m}\right)$.

The multiplication in $G F\left(2^{m}\right)$ was usually a very expensive operation. The 64 bit carry-less multiplier, which is now available on various x86 platforms, significantly accelerates the multiplication in binary fields. In this work we use both explicit and recursive versions of Karatsuba-Ofman for different sizes. We chose explicit forms for 2-, 3- and 5-term Karatsuba and recursive forms for 4-,7and 9-terms, balancing performance gain against code size. For a comparison of Karatsuba implementations with vector extensions see [18] by Su and Fan.

For the inversion in $G F\left(2^{m}\right)$ we use the Itoh-Tsujii Algorithm(ITA) 11. The ITA performs the inversion with exactly $m-1$ squares and $\left\lfloor\log _{2}(m-1)\right\rfloor+$ $H(m-1)-1$ multiplications with Hamming weight $H$. The general recursive formula to obtain the chain can be given as

$$
\begin{aligned}
& 1+2^{n}+2^{2 n}+\ldots+2^{(k-2) n}= \\
& \left\{\begin{array}{lll}
\left(1+2^{n}\right) \times\left(1+2^{2 n}+\ldots+2^{(k-3) n}\right), & \text { if } k-1 \equiv 0 & \bmod 2 \\
1+\left(2^{n} \times\left(1+2^{n}\right) \times\left(1+2^{2 n}+\ldots+2^{(k-4) n}\right),\right. & \text { if } k-1 \equiv 1 & \bmod 2
\end{array}\right.
\end{aligned}
$$

The running time boundaries for this algorithm are mainly determined by squares and requires a serious amount of time. In this implementation, fortunately, the costly inversion is only required once per point multiplication.

\subsection{Reduction}

The reduction is a very crucial part of the implementation. The two most important field operations, squaring and multiplication, both require a reduction with the field polynomial $f(x)$, which can be seen as a multiplication with the reduction polynomial. In this work we deal with the NIST polynomials and two polynomials from the SECG standard[17], either trinomials or pentanomials. In this section, we will describe our strategy for each type and discuss special opportunities to speed up the reduction schemes with AVX. 
The reduction is basically a multiplication of the upper half of the double sized element with the reduction polynomial. Let $f(x)=x^{m}+r(x)$ with degree $\operatorname{deg}(r)=k$ be the reduction polynomial over $G F\left(2^{m}\right)$. We split the polynomial $c(x)=c_{H}(x)+c_{L}(x)$ with degree $\operatorname{deg}(c)=m+t$ into two parts where

$$
c_{H}(x)=\sum_{i=m}^{t} c_{i} x^{i} \quad \text { and } \quad c_{L}(x)=\sum_{i=0}^{m-1} c_{i} x^{i} .
$$

The reduction of $c(x)$ is then computed as

$$
c^{\prime}(x)=c_{L}(x) \oplus c_{H}(x) \cdot r(x) .
$$

The resulting polynomial $c^{\prime}(x)$ has degree $d=\operatorname{deg}\left(c^{\prime}\right)=\max (m-1, t+k)$. In case of $d \geq m$, the reduction is applied recursively.

"Left-to-Right In-Place Shift and Add" approach is the consequence. Since the multiplication of a word by one bit is a simple shift, this multiplication can be applied as a series of shifts and adds. This makes especially sense, if we remember that the amount of bits in $r(x)$ is very small (here either 2 or 4). In our experiments, this technique produced better results than other reduction schemes such as Barrett's method [12] 13].

The reduction for trinomials is very straightforward - we successively shift the top word by the components of $r(x)$ to the right and add the result to the remainder, while skipping multiples of 64 and 128 bit with memory alignments. In order to avoid duplication of work, one should execute the shift and add from the left to the right and adding the current word immediately to the remainder. Under the assumption that we are about to reduce a double quadword $w$ by component $x^{k}$, we shift $w$ exactly $(m-k \bmod 64)$ bits to the right and add it to the remainder while skipping $l \times 128$ bits, where $l=\left\lfloor\frac{m-k}{128}\right\rfloor$. If $(m-k \bmod 128) \geq$ 64 , however, we need to add the lower half of $w$ to the previous word.

The strategy for pentanomials differs from the trinomial way in terms of recombination. Instead of directly adding each component to the remainder, we gather the results of left and right shifts of a vector A in separated vectors and recombine them by finally adding them to the remainder, which saves three alignments per 128 bit element. Figure 1 shows the combination steps for a vector A.

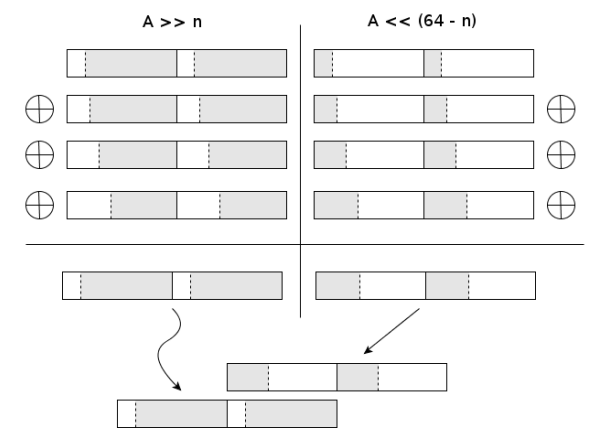

Fig. 1: Example step of the re-combination strategy for pentanomials 
Fast vector shifting uses the (faster) 128 bit shift instruction(pslldq/psrldq), which is feasible whenever the difference of two components in the polynomial is a multiple of 8 . With the polynomials from 1 , we can apply this trick for the polynomial $f(x)=x^{163}+x^{7}+x^{6}+x^{3}+1$ where $163-3=160$. Additionally, we have the components $x^{10}$ and $x^{2}$ in the reduction polynomial for $G F\left(2^{571}\right)$ but here the fast shifting interferes with the recombination strategy, where the savings for the alignment pay off in comparison.

Special reduction schemes are possible in $G F\left(2^{283}\right)$ and necessary in $G F\left(2^{239}\right)$. A fast reduction in $G F\left(2^{283}\right)$ was proposed in [1] and uses the observation $f(x)=x^{283}+x^{12}+x^{7}+x^{5}+1=x^{283}+\left(x^{7}+1\right) \cdot\left(x^{5}+1\right)$.

The polynomial $f(x)=x^{239}+x^{158}+1$ is one of the two polynomials suggested in [17] for this field size and currently integrated in OpenSSL. For the implementation with vector extensions, this polynomial is suboptimal. This is caused by the small distance $239-158=81<128$, which means that a 128 bit word of the remainder affects itself during the reduction, which makes shift and add reduction inefficient for this size. The $G F\left(2^{239}\right)$ reduction is therefore provided in 64 bit mode only. When using vector extensions, the polynomial $f(x)=x^{239}+x^{36}+1$ (which has also been suggested by SECG) is much faster. Another approach would be to implement the reduction with a special version of Barrett's method, which should increase the performance of this field significantly. Due to this issues we do not recommend the use of this field. Reduction in $G F\left(2^{233}\right)$ is much faster and this particular curve has almost the same security level (see 1) with an only slightly shorter key. Anyhow, we added this curve to our implementation for convenience.

\subsection{Mul\&Add reduction}

The pclmulqdq instruction can be utilized for reduction. Thus, each 64 bit word of the operand $c_{H}(x)$ must be multiplied with $r(x)=\sum_{i=0}^{k} r_{i} x^{i}$ and added to the remainder $c_{L}(x)$. Obviously, $k$ must hold the condition $k<64$ to ensure the lowest number of multiplications, which is exactly $t=\left\lceil\frac{\log _{2}\left(c_{H}(x)\right)}{64}\right\rceil$ then. If $k>63$, we must multiply each word more than once, particularly $s=\left\lceil\frac{k}{64}\right\rceil$ times per word and we therefore require $t \cdot s$ multiplications overall.

In order to spare the additional reduction step, as described above, the multiplications are executed from the left to the right and the results are immediately added to the remainder. Due to the fact that the bits of $c(x)$ are results from either multiplication or squaring and are stored into a series of words where the border between $c_{L}(x)$ and $c_{H}(x)$ at position $m-1$ is right in the middle of a 64 bit block, each multiplication result must be aligned in order to be added to the remainder. Therefore, we can either initially shift the polynomial $r(x)$ to the left by $l=m$ mod 64 bits (if $k+l<64$ still holds), or we can shift the input words $l$ bits to the right, or $64-l$ bits to the left. Thereby, the operand needs to be saved temporarily as the reduction is performed in-place, requiring more temporary variables. Shifting the operand especially makes sense if the data in 
$c_{H}(x)$ overlaps into an additional register, thus requiring $t+1$ multiplications. For example the reduction in $G F\left(2^{163}\right)$, the bits of $c_{H}(x)=\sum_{i=163}^{325} c_{i} x^{i}$ are spread over four 64 bit words. For our target curves, the operand shifting requires to much efforts compared to a single multiplication on Haswell, whereas on the Sandy/Ivy Bridge this shifting pays off. Obviously, the proposed technique makes more sense the greater the hamming weight of $r(x)$ is, and if all the bits in $r(x)$ are in one 64 bit word $(s=1)$. Fortunately, this is the case for the NIST curves over $G F\left(2^{163}\right), G F\left(2^{283}\right)$ and $G F\left(2^{571}\right)$.

As a matter of fact, the performance of the pclmulqdq reduction strongly depends on the underlying architecture. This reduction scheme starts to pay off when the multiplier performs two multiplications faster than the according shifts and additions. Let the reduction polynomial be pentanomial with no special properties as fast shifts or other special properties. For the "shift" step of the shift\&add reduction, the required amount of operations for each 128 bit word is $4 \times 2$ SHIFT's (psllq/psrlq) plus $3 \times 2$ XOR's. Requiring the same amount of operations for the "add" step, the multiplier must be able to perform 2 multiplications faster than this in order to beat the shift\&add implementation for pentanomial reduction polynomials. On the Sandy/Ivy Bridge, this cannot be performed faster than 10 cycles (usually more), whereas one 64 bit multiplication has a latency of 14 cycles. This explains why this reduction scheme does not pay off on these architectures.

On Haswell however, with a latency of 7 and a throughput of only 2 cycles, this can be performed in 9 cycles. In many cases, one can make advantage of the small throughput by fetching multiple multiplications at once to gain even better performance.

\section{Results}

This section presents the results for the binary field arithmetic and shows the impact of the improved LD/Montgomery point multiplication and the resulting performance gain for both ECDH and ECDSA of the OpenSSL implementation. The corresponding tables can be found in the appendix. Since our implementation is suitable for several architectures, we provide data for a Haswell Core i7-4770 CPU at 3.40GHz (HSW) and a Core i5-3210M Ivy Bridge (IVB), running with $2.50 \mathrm{GHz}$. Tests on a Sandy Bridge processor have produced very similar relative results as on the Ivy Bridge. To reduce randomness, we followed the guidelines from ECRYPT Benchmarking of Cryptographic Systems(eBACS) 20. The code was compiled with the latest gcc in version 4.8.1. As tools for measurement we used the OpenSSL built-in speed tool as well as a cycle counter using the RDTSC instruction, similar to [10, with slightly different repetition factors. The OpenSSL speed utility can be used to reproduce our results after applying the patch and indicates the patch's impact to the overall performance of OpenSSL ECDH and ECDSA operations. 
Binary field arithmetic costs are shown in Table 5 . The results indicate the benefits of the fast pclmulqdq on Haswell and the performance difference of squaring and multiplication.

Reduction costs can be found in Table 6 on the different architectures. It shows the performance difference of trinomials and pentanomials as well as the performance loss due to the 64 bit mode in $G F\left(2^{239}\right)$. Furthermore, we see the mul\&add reduction outperforming the shift\&add reduction with factor 1.25 in $G F\left(2^{163}\right)$ and about 1.62 in $G F\left(2^{283}\right)$ and 1.61 in $G F\left(2^{571}\right)$ on Haswell. However, the shift\&add implementation does not use AVX2 features, which could eventually allow improvements.

The computational costs for side channel protection by hiding the coordinates in order to assure a fixed memory access pattern per round are also not neclectable. A single data veiling requires twelve logical operations at the cost of an XOR. Additionally, one requires four temporary variables in order to hide the data processing for all four coordinates involved in the point multiplication. This has of course some performance impact to the point multiplication process, as this performance overhead applies for each loop execution.

The point multiplication's importance has been pointed out several times by now. Table 7 shows the results for $k \cdot P$ in cycles. We find that the amount of cycles has been substantially diminished, up to a factor of almost 12. Also, we observe the impact of the improved code flow for the Koblitz rather than random curves, which is noticeable in both absolute numbers and the relative speedup. Hereby, we have to mention that the numbers for the unpatched OpenSSL-1.0.1e are not constant, since it is not a constant time implementation, especially the bit length of $k$ is not fixed. Therefore, the speedup factor is also not consistent and will differ from run to run.

Elliptic curve DiffieHellman operations are presented in Table 11 and Figure 2, measured with the OpenSSL speed tool and therefore the curve identifiers have been changed to nistk for Koblitz and nistb for random curves. Since the ECDH is basically a point multiplication, the speedup is very high. Prime curves are currently more frequently used in server implementations. As demonstrated in Table 8 our improvements to the point multiplication of the binary curves result in a big performance lead.


Fig. 2: ECDH - operations per second. OpenSSL (with enable-ec_nistp_64_gcc_128 flag) versus proposed implementation on the Haswell processor 
Elliptic curve signature and verification algorithms have considerably more overhead than the ECDH. However, as shown in Tables 9 and 10, we perceive an average speedup of factor 4.9 (sign) and 6.2 (verify) on Ivy Bridge as well as 4.5 and 6.3 on Haswell, respectively. Since the bit length for the ECDSA in OpenSSL has been fixed before the point multiplication in response to a timing attack [7, the resulting scalar $k$ is not in the interval $[1, n]$ anymore and is either one or two bits longer (but constant for a specific field) than the original scalar (depending on the form of the corresponding curve order $n$ ), which slightly increases the amount of cycles for the patched version.

\section{Comparison to other works}

Most works about the implementation of binary elliptic curves with the use of vector instructions are aiming to break speed records and thus often exploit algebraic properties. This work aims for provision of a generic point multiplication for the well known NIST/SECG curves over the binary field, without any compromises in regard to security. This work does not use precomputation schemes (such as wNAF, $\tau \mathrm{NAF}$ ), point halving, special fields with beneficial properties (such as $\mathbb{F}_{q^{2}}$ ), or other algebraic properties (e.g., Forbenius morphism).

In Table 4 we show results for implementations of random point multiplication for binary elliptic curves in NIST/SEC fields. All numbers are given in $10^{3}$ cycles on a single core.

\begin{tabular}{|c|c|c|c|c|c|c|c|c|}
\hline & Work & $\mathbf{S C P}^{1}$ & CPU & Method & $2^{233}$ & $2^{283}$ & $2^{409}$ & $2^{571}$ \\
\hline $\begin{array}{l}N \\
\stackrel{1}{0} \\
0 \\
1\end{array}$ & $\begin{array}{l}\text { Aranha et al. } 2 \\
\text { Taverne et al. } 19 \\
\text { Aranha et al. } 3 \\
\text { This work }\end{array}$ & $\begin{array}{c}\text { no } \\
\text { no } \\
\text { no } \\
\text { yes } \\
\text { yes }\end{array}$ & $\begin{array}{l}\text { Core i7 }-860 \\
\text { Core i7 }(\mathrm{SNB}) \\
\text { Core i7 }(\mathrm{SNB}) \\
\text { Core i5 (IVB) } \\
\text { Core i7 (HSW) }\end{array}$ & $\begin{array}{l}\text { 4-TNAF } \\
5-\tau \text { NAF, } \tau \& \text { add } \\
5-\tau \text { NAF, } \tau \text { Ł283/2」 } \\
\text { LD-Montgomery } \\
\text { LD-Montgomery }\end{array}$ & $\begin{array}{r}- \\
068 \\
- \\
128 \\
81\end{array}$ & $\begin{array}{r}386 \\
- \\
099 \\
217 \\
118\end{array}$ & $\begin{array}{r}- \\
264 \\
- \\
511 \\
286\end{array}$ & $\begin{array}{r}1656 \\
- \\
- \\
1095 \\
566\end{array}$ \\
\hline 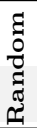 & $\begin{array}{l}\text { Aranha et al. } 2 \\
\text { Taverne et al. } 19 \\
\text { This work }\end{array}$ & $\begin{array}{l}\text { no } \\
\text { no } \\
\text { yes } \\
\text { yes }\end{array}$ & $\begin{array}{l}\text { Core i7-860 } \\
\text { Core i7 (SNB) } \\
\text { Core i5 (IVB) } \\
\text { Core i7 (HSW) }\end{array}$ & $\begin{array}{l}\text { LD-Montgomery } \\
4-\omega \text { NAF } \\
\text { LD-Montgomery } \\
\text { LD-Montgomery }\end{array}$ & $\begin{array}{r}- \\
180 \\
148 \\
91\end{array}$ & $\begin{array}{r}793 \\
- \\
254 \\
135\end{array}$ & $\begin{array}{r}- \\
738 \\
599 \\
325\end{array}$ & $\begin{array}{r}4440 \\
- \\
1271 \\
650\end{array}$ \\
\hline
\end{tabular}

Table 4: Unknown Point Multiplication over NIST Curves (Koblitz)

This work yields very good results for random curves and wins against all current state-of-the-art implementations over the NIST fields, even with the costly side channel countermeasures. Since all previous works have been implemented on the Sandy/Ivy Bridge, we compare with the Ivy Bridge implementation here. For the random curve over the $G F\left(2^{233}\right)$ NIST field, our implementation is about factor $1.22\left(1.23\right.$ in $\left.G F\left(2^{409}\right)\right)$ faster than [19] and even factor $3\left(G F\left(2^{283}\right)\right)$ and $3.5\left(G F\left(2^{571}\right)\right)$ faster than reported in [2] for a single core. Although our Ivy Bridge implementation for Koblitz curves beats the numbers presented in 2 by $1.77 \mathrm{x}$ and $1.51 \mathrm{x}, 19$ achieve results which are factor 1.53 faster, whilst [3] is even as twice as fast.

\footnotetext{
${ }^{1}$ Side Channel Protection
} 
For further comparison, we give the latest numbers of protected (as claimed by the authors) point multiplication implementations at the 128 bit security level, using curves with special algebraic properties. Bos et al. [6] report 117,000 cycles for a protected point multiplication on a Kummer surface with the Montgomery ladder and [16] reports 115,000 cycles on a GLS curve using 2-GLV (double\&add) on a single core. At the same security level, this work achieves a point multiplication in 118,000 cycles with the OpenSSL patch 5 on the Haswell processor, without using special fields, exploiting algebraic properties or precomputation techniques. At the 256 bit security level, this work achieves a EC point multiplication in about 566,000 (Koblitz) and 650,000 (random) cycles.

\section{Conclusion}

In this work, we proposed a fast, constant time implementation of the point multiplication on binary elliptic curves at a security level of 80 bits and greater, standardized by NIST and SECG.

We analyzed major implementation threats for server environments and secured the implementation by applying countermeasures against common and dangerous side channel threats such as cache and remote timing attacks. Over the last years, several publications have pointed out that these side channel attacks are more than a theoretical but a serious menace. To address this issue, we provide a constant time implementation of the elliptic curve point multiplication and binary field arithmetic, without key dependent branches and a fixed memory access pattern.

Additionally, we introduced an improved version for the well known LD/Montgomery multiplication with enhancements on the arithmetic level, provided a patch for the OpenSSL library and showed its significant performance impact. This improvement is available on all 64 bit architectures implementing at least SSE4 (better: AVX) and the pclmulqdq instruction. The implementation has been contributed to OpenSSL as patch for version 1.0.1e and is available for download [5].

We further showed that this impact is not limited to the point multiplication itself but improves the servers performance regarding ECDH operations and signature based cryptography. This further leads to a major performance improvement for servers and clients conducting TLS handshakes. This is an essential enhancement especially for server environments, which are often required to conduct a big number of handshakes simultaneously. Also, our results might indicate a different view to the binary ECC on server architectures. 


\section{References}

1. D. Aranha, A. Faz-Hernndez, J. Lpez, and F. Rodrguez-Henrquez, Faster implementation of scalar multiplication on koblitz curves, in Progress in Cryptology LATINCRYPT 2012, A. Hevia and G. Neven, eds., vol. 7533 of Lecture Notes in Computer Science, Springer Berlin Heidelberg, 2012, pp. 177-193.

2. D. Aranha, J. LPEZ, AND D. HANkERson, Efficient software implementation of binary field arithmetic using vector instruction sets, in Progress in Cryptology LATINCRYPT 2010, M. Abdalla and P. Barreto, eds., vol. 6212 of Lecture Notes in Computer Science, Springer Berlin Heidelberg, 2010, pp. 144-161.

3. D. F. Aranha, A. Faz-Hernndez, J. Lpez, and F. Rodrguez-Henrquez, Faster implementation of scalar multiplication on koblitz curves. Cryptology ePrint Archive, Report 2012/519, 2012. http://eprint.iacr.org/.

4. D. F. Aranha, J. López, And D. Hankerson, Efficient Software Implementation of Binary Field Arithmetic Using Vector Instruction Sets, in The First International Conference on Cryptology and Information Security (LATINCRYPT 2010), M. Abdalla and P. S. L. M. Barreto, eds., vol. 6212 of LNCS, 2010, pp. 144-161.

5. M. Bluhm And S. Gueron, A fast vectorized implementation of binary elliptic curves on $x 86-64$ processors, 2013. http://rt.openssl.org/Ticket/Display. html?id=3117.

6. J. W. Bos, C. Costello, H. Hisil, And K. Lauter, Fast cryptography in genus 2. Cryptology ePrint Archive, Report 2012/670, 2012. http://eprint.iacr.org/.

7. B. B. Brumley AND N. Tuveri, Remote timing attacks are still practical, in Proceedings of the 16th European conference on Research in computer security, ESORICS'11, Berlin, Heidelberg, 2011, Springer-Verlag, pp. 355-371.

8. P.-A. Fouque, D. Ral, F. Valette, And M. Drissi, The carry leakage on the randomized exponent countermeasure, in Cryptographic Hardware and Embedded Systems CHES 2008, E. Oswald and P. Rohatgi, eds., vol. 5154 of Lecture Notes in Computer Science, Springer Berlin Heidelberg, 2008, pp. 198-213.

9. S. Gueron And M. KounAvis, Intel carry-less multiplication instruction and its usage for computing the gcm mode, April 2008. http://software.intel.com/ sites/default/files/article/165685/clmul-wp-rev-2.01-2012-09-21.pdf

10. S. Gueron AND V. KRAsnov, Parallelizing message schedules to accelerate the computations of hash functions, Journal of Cryptographic Engineering, 2 (2012), pp. 241-253.

11. T. Iтон AND S. Tsujis, A fast algorithm for computing multiplicative inverses in gf(2m) using normal bases, Inf. Comput., 78 (1988), pp. 171-177.

12. M. Knezevic, K. Sakiyama, J. Fan, And I. Verbauwhede, Modular reduction in $g f\left(2^{n}\right)$ without pre-computational phase, in WAIFI, 2008, pp. 77-87.

13. A. O. Krzysztof Jankowski, Pierre Laurent, Intel polynomial multiplication instruction and its usage for elliptic curve cryptography, 2012.

14. J. LPEZ AND R. DAHAB, Fast multiplication on elliptic curves over gf(2m) without precomputation, in Cryptographic Hardware and Embedded Systems, . Ko and C. Paar, eds., vol. 1717 of Lecture Notes in Computer Science, Springer Berlin Heidelberg, 1999, pp. 316-327.

15. P. L. Montgomery, Speeding the Pollard and Elliptic Curve Methods of Factorization, Mathematics of Computation, 48 (1987), pp. 243-264.

16. T. Oliveira, J. Lpez, D. F. Aranha, and F. Rodrguez-Henrquez, Two is the fastest prime. Cryptology ePrint Archive, Report 2013/131, 2013. http:// eprint.iacr.org/ 
17. Standards for Efficient Cryptography Group, SEC 2: Recommended Elliptic Curve Domain Parameters, in Standards for Efficient Cryptography, 2000.

18. C. SU AND H. FAN, Impact of intel's new instruction sets on software implementation of $g f(2)[x]$ multiplication, Inf. Process. Lett., 112 (2012), pp. 497-502.

19. J. Taverne, A. Faz-Hernndez, D. Aranha, F. Rodrguez-Henrquez, D. HanKERSON, AND J. LPEZ, Speeding scalar multiplication over binary elliptic curves using the new carry-less multiplication instruction, Journal of Cryptographic Engineering, 1 (2011), pp. 187-199.

20. E. VAMPIRE, Ecrypt benchmarking of cryptographic systems. http://bench.cr. yp.to/supercop.html, 2013.

21. D. WEBER AND T. DENNY, The solution of mccurley's discrete log challenge, in Advances in Cryptology CRYPTO '98, H. Krawczyk, ed., vol. 1462 of Lecture Notes in Computer Science, Springer Berlin Heidelberg, 1998, pp. 458-471.

\section{A.1 Speed results}

\begin{tabular}{c||c|c||c|c||r|r}
\multirow{2}{*}{ Field } & \multicolumn{2}{c}{ Squaring } & \multicolumn{2}{c||}{ Multiplication } & \multicolumn{2}{c}{ Inversion } \\
& HSW & IVB & HSW & IVB & HSW & IVB \\
\hline$G F\left(2^{163}\right)$ & 21 & 21 & 34 & 71 & 4,271 & 4,811 \\
$G F\left(2^{193}\right)$ & 17 & 19 & 37 & 98 & 4,122 & 4,788 \\
$G F\left(2^{233}\right)$ & 18 & 21 & 38 & 87 & 6,074 & 6,956 \\
$G F\left(2^{239}\right)$ & 49 & 44 & 67 & 100 & 15,349 & 14,777 \\
$G F\left(2^{283}\right)$ & 27 & 30 & 53 & 121 & 11,688 & 9,677 \\
$G F\left(2^{409}\right)$ & 28 & 31 & 97 & 227 & 15,182 & 15,648 \\
$G F\left(2^{571}\right)$ & 61 & 68 & 158 & 335 & 37,118 & 42,595 \\
\hline
\end{tabular}

Table 5: Cycles for arithmetic operations in $G F\left(2^{m}\right)$ (without addition)

\begin{tabular}{c||c|c||c|c} 
Field & $\begin{array}{c}\text { Shift\&Add } \\
\text { (Haswell) }\end{array}$ & $\begin{array}{c}\text { Mul\&Add } \\
\text { (Haswell) }\end{array}$ & $\begin{array}{c}\text { Shift\&Add } \\
\text { (Ivy Bridge) }\end{array}$ & $\begin{array}{c}\text { Mul\&Add } \\
\text { (Ivy Bridge) }\end{array}$ \\
\hline$G F\left(2^{163}\right)$ & 15 & $\mathbf{1 2}$ & 18 & 31 \\
$G F\left(2^{193}\right)$ & $\mathbf{1 1}$ & - & 13 & - \\
$G F\left(2^{233}\right)$ & $\mathbf{1 2}$ & - & 15 & - \\
$G F\left(2^{239}\right)$ & 42 & - & $\mathbf{4 1}$ & - \\
$G F\left(2^{283}\right)$ & 21 & $\mathbf{1 3}$ & 25 & 57 \\
$G F\left(2^{409}\right)$ & $\mathbf{1 5}$ & - & 20 & - \\
$G F\left(2^{571}\right)$ & 37 & $\mathbf{2 3}$ & 48 & 81 \\
\hline
\end{tabular}

Table 6: Comparison of the two reduction schemes on Haswell and Ivy Bridge 


\begin{tabular}{c||rr|rr||rr}
\multicolumn{1}{c||}{$\begin{array}{c}\text { Binary } \\
\text { Curve }\end{array}$} & \multicolumn{2}{c|}{ OpenSSL-1.0.1e } & \multicolumn{2}{c|}{ OpenSSL patch } & \multicolumn{2}{c}{ Speedup factor } \\
Ivy Bridge & Haswell & Ivy Bridge & Haswell & Ivy Bridge & Haswell \\
\hline & & & & & & \\
sect163k1 & 624,949 & 543,097 & 66,378 & 45,642 & 9.42 & 11.90 \\
sect163r1 & 643,236 & 563,928 & 76,534 & 49,869 & 8.40 & 11.31 \\
sect163r2 & 646,374 & 567,854 & 77,238 & 50,127 & 8.37 & 11.33 \\
sect193r1 & 801,932 & 572,307 & 121,127 & 73,296 & 6.62 & 7.81 \\
sect193r2 & 818,985 & 567,929 & 121,163 & 73,442 & 6.76 & 7.73 \\
sect233k1 & 993,195 & 681,347 & 128,474 & 81,133 & 7.73 & 8.40 \\
sect233r1 & $1,030,043$ & 712,864 & 148,230 & 91,372 & 6.95 & 7.80 \\
sect239k1 & $1,027,541$ & 718,156 & 185,629 & 144,816 & 5.54 & 4.96 \\
sect283k1 & $1,892,016$ & 125,8711 & 217,192 & 118,120 & 8.71 & 10.66 \\
sect283r1 & $2,019,134$ & $1,341,366$ & 254,163 & 134,571 & 7.94 & 9.97 \\
sect409k1 & $3,315,895$ & $1,984,879$ & 510,787 & 286,090 & 6.49 & 6.94 \\
sect409r1 & $3,655,279$ & $2,142,064$ & 599,002 & 325,279 & 6.10 & 6.59 \\
sect571k1 & $7,659,288$ & $4,764,870$ & $1,094,765$ & 566,257 & 7.00 & 8.41 \\
sect571r1 & $8,432,393$ & $5,222,559$ & $1,270,666$ & 659,528 & 6.64 & 7.92
\end{tabular}

Table 7: Point multiplication results in comparison to OpenSSL-1.0.1e in cycles

\begin{tabular}{|c|c|c|c|c|}
\hline \multirow{2}{*}{$\begin{array}{l}\text { Curve } \\
\text { Identifier }\end{array}$} & \multicolumn{2}{|c|}{ Patched OpenSSL-1.0.1e } & \multicolumn{2}{|c|}{ Speedup factor } \\
\hline & Ivy Bridge & Haswell & Ivy Bridge & Haswell \\
\hline secp160r 1 & 4444.3 & 7391.5 & - & - \\
\hline nistk163 & 27837.0 & 67212.4 & 6.26 & 9.09 \\
\hline nistb163 & 24043.5 & 61667.8 & 5.41 & 8.34 \\
\hline nistp224 & 7573.1 & 11993.8 & - & - \\
\hline nistk233 & 14946.4 & 39102.2 & 1.97 & 3.26 \\
\hline nistb233 & 13057.0 & 35246.4 & 1.72 & 2.94 \\
\hline nistp256 & 3891.5 & 6489.0 & - & - \\
\hline nistk283 & 9026.5 & 27586.5 & 2.32 & 4.25 \\
\hline nistb283 & 7754.4 & 24320.7 & 1.99 & 3.75 \\
\hline nistp384 & 1051.9 & 1848.5 & - & - \\
\hline nistk409 & 3879.5 & 11611.2 & 3.69 & 6.28 \\
\hline nistb409 & 3319.6 & 10238.1 & 3.16 & 5.54 \\
\hline nistp521 & 971.0 & 1682.8 & - & - \\
\hline nistk571 & 1822.3 & 5941.8 & 1.88 & 3.53 \\
\hline nistb571 & 1565.0 & 5158.8 & 1.61 & 3.07 \\
\hline
\end{tabular}

Table 8: Comparison of ECDH operations per second for binary and prime elliptic curves on Ivy Bridge and Haswell 




Table 9: ECDSA sign and verify cycles for the NIST curves on Ivy Bridge and Haswell 


\begin{tabular}{|c|c|c|c|c|c|c|c|}
\hline & Binary & OpenSSI & $-1.0 .1 e$ & OpenSS & patch & Speedup & factor \\
\hline & Curve & Ivy Bridge & Haswell & Ivy Bridge & Haswell & Ivy Bridge & Haswell \\
\hline & nistk163 & $3,749.9$ & $6,465.3$ & $17,721.8$ & $36,872.6$ & 4.73 & 5.70 \\
\hline & nistk233 & $1,881.7$ & $3,259.2$ & $10,359.0$ & $22,998.4$ & 5.51 & 7.06 \\
\hline & nistk283 & $1,267.5$ & $2,204.7$ & $6,688.9$ & $16,884.9$ & 5.28 & 7.66 \\
\hline & nistk409 & 542.2 & 977.0 & $3,140.9$ & $8,150.0$ & 5.79 & 8.34 \\
\hline Z & nistk571 & 257.6 & 466.4 & $1,556.0$ & $4,424.1$ & 6.04 & 9.49 \\
\hline 次 & nistb163 & $3,766.5$ & $6,487.3$ & $16,203.5$ & $35,110.0$ & 4.30 & 5.41 \\
\hline & nistb233 & $1,893.1$ & $3,279.2$ & $9,386.5$ & $21,468.8$ & 4.96 & 6.55 \\
\hline & nistb283 & $1,265.7$ & $2,196.4$ & $5,962.3$ & $15,602.7$ & 4.71 & 7.10 \\
\hline & nistb409 & 539.3 & 976.3 & $2,763.4$ & $7,423.1$ & 5.12 & 7.60 \\
\hline & nistb571 & 257.2 & 466.6 & $1,354.8$ & $3,977.0$ & 5.27 & 8.52 \\
\hline & nistk163 & $1,578.6$ & $3,159.5$ & $11,688.1$ & $26,508.4$ & 7.40 & 8.39 \\
\hline & nistk233 & $1,211.6$ & $2,419.8$ & $6,439.4$ & $15,557.1$ & 5.31 & 6.43 \\
\hline & nistk283 & 639.3 & $1,355.7$ & $3,951.1$ & $11,003.2$ & 6.18 & 8.12 \\
\hline & nistk409 & 361.9 & 839.1 & $1,757.1$ & $4,845.0$ & 4.86 & 5.77 \\
\hline 后 & nistk571 & 159.9 & 368.3 & 834.6 & $2,533.6$ & 5.22 & 6.88 \\
\hline 至 & nistb163 & $1,514.5$ & $3,043.9$ & $10,453.8$ & $24,904.8$ & 6.90 & 8.18 \\
\hline & nistb233 & $1,150.4$ & $2,348.0$ & $5,711.9$ & $14,095.6$ & 4.97 & 6.00 \\
\hline & nistb283 & 594.2 & $1,283.5$ & $3,445.5$ & $9,888.5$ & 5.80 & 7.70 \\
\hline & nistb409 & 344.2 & 786.9 & $1,522.4$ & $4,361.9$ & 4.42 & 5.54 \\
\hline & nistb571 & 145.7 & 341.0 & 724.9 & $2,251.6$ & 4.98 & 6.60 \\
\hline
\end{tabular}

Table 10: OpenSSL ECDSA sign and verification operations per second for the NIST curves on Ivy Bridge and Haswell

\begin{tabular}{c||rr|rr||rr}
\multicolumn{1}{c||}{$\begin{array}{c}\text { Binary } \\
\text { Curve }\end{array}$} & \multicolumn{2}{c|}{ OpenSSL-1.0.1e } & \multicolumn{2}{c||}{ OpenSSL patch } & \multicolumn{2}{c}{ Speedup factor } \\
& Ivy Bridge & \multicolumn{1}{c}{ Haswell } & Ivy Bridge & Haswell & Ivy Bridge & Haswell \\
\hline & & & & & & \\
nistk163 & $4,129.0$ & $6,586.9$ & $34,739.0$ & $67,029.6$ & 8.41 & 10.18 \\
nistk233 & $2,628.4$ & $5,121.9$ & $17,013.4$ & $39,441.3$ & 6.47 & 7.70 \\
nistk283 & $1,338.5$ & $2,825.7$ & $9,017.6$ & $27,718.5$ & 6.74 & 9.81 \\
nistk409 & 836.3 & $1,745.8$ & $3,884.0$ & $11,634.2$ & 4.64 & 6.66 \\
nistk571 & 406.5 & 763.2 & $1,824.1$ & $5,930.9$ & 4.49 & 7.77 \\
& & & & & & \\
nistb163 & $3,217.6$ & $6,382.5$ & $24,237.2$ & $60,729.6$ & 7.53 & 9.52 \\
nistb233 & $2,443.5$ & $4,881.9$ & $13,114.3$ & $35,230.4$ & 5.37 & 7.22 \\
nistb283 & $1,244.3$ & $2,651.6$ & $7,711.6$ & $24,456.4$ & 6.20 & 9.22 \\
nistb409 & 723.4 & $1,640.3$ & $3,382.3$ & $10,228.6$ & 4.68 & 6.24 \\
nistb571 & 368.3 & 693.8 & $1,943.5$ & $5,172.1$ & 5.28 & 7.45 \\
& & & & & & \\
\hline
\end{tabular}

Table 11: OpenSSL ECDH operations per second over the NIST curves 


\section{A.2 New 2P-Algorithm subfunctions}
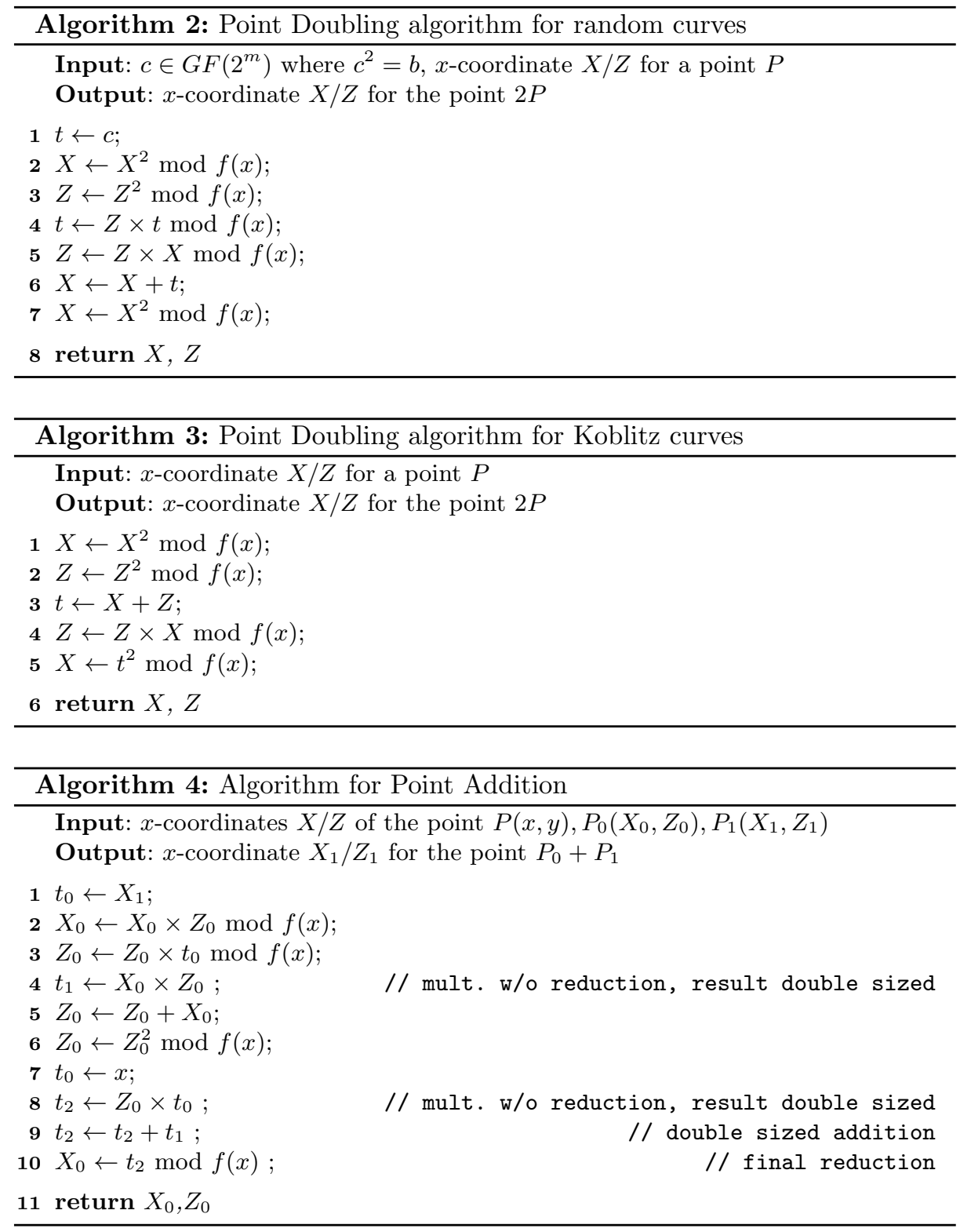